\title{
Congenital diaphragmatic hernia as a part of Nance-Horan syndrome?
}

\author{
Molka Kammoun ${ }^{1} \cdot$ Paul Brady $^{1} \cdot$ Luc De Catte $^{2} \cdot$ Jan Deprest ${ }^{2} \cdot$ Koenraad Devriendt $^{1}{ }^{1} \cdot$ Joris Robert Vermeesch $^{1}$
}

Received: 20 March 2017 / Revised: 26 September 2017 / Accepted: 17 October 2017 / Published online: 22 January 2018

(c) European Society of Human Genetics 2018

\begin{abstract}
Nance-Horan syndrome is a rare X-linked developmental disorder characterized by bilateral congenital cataract, dental anomalies, facial dysmorphism, and intellectual disability. Here, we identify a patient with Nance-Horan syndrome caused by a new nonsense $N H S$ variant. In addition, the patient presented congenital diaphragmatic hernia. NHS gene expression in murine fetal diaphragm was demonstrated, suggesting a possible involvement of $N H S$ in diaphragm development. Congenital diaphragmatic hernia could result from NHS loss of function in pleuroperitoneal fold or in somites-derived muscle progenitor cells leading to an impairment of their cells migration.
\end{abstract}

\section{Introduction}

Congenital diaphragmatic hernia $(\mathrm{CDH})$ is a life-threatening condition affecting 2.3 per 10,000 births [1]. The malformation results from a defect of the muscular or of the tendinous portion of the diaphragm leading the abdominal viscera to herniate into the thoracic cavity and to compete for space with the developing lungs. Clinically, $\mathrm{CDH}$ occurs either as an isolated malformation in approximately half of the cases [1,2], or in association with other malformations. Cardiovascular defects are by far the most common associated malformations. Less frequently, $\mathrm{CDH}$ is associated with musculoskeletal, urogenital, or brain malformations [1, $3,4]$, and is rarely associated with ocular defects that affect about $1 \%$ of cases $[1,4]$. As summarized in Table 1, CDHassociated congenital eye malformations consist mainly of anophthalmia and microphthalmia in conjunction with colobomas. Congenital cataract and late onset cataract have been described with $\mathrm{CDH}$ in one case of HCCS deletion [5] and in a few cases of $L R P 2$ mutation $[6,7]$, respectively.

The genetics of $\mathrm{CDH}$ is still poorly understood. For isolated $\mathrm{CDH}$, a chromosomal rearrangement is identified in

Joris Robert Vermeesch

joris.vermeesch@uzleuven.be

1 Department of Human Genetics, KU Leuven, O\&N I Herestraat 49, box 602, 3000 Leuven, Belgium

2 Department Obstetrics and Gynecology, University Hospital Leuven, Leuven, Belgium
$10 \%$ of cases [21]. In addition, $\mathrm{CDH}$ genetics is highly heterogeneous with at least 70 known $\mathrm{CDH}$ causal genes [2], making whole exome sequencing an attractive approach for $\mathrm{CDH}$ genetic causes identification. This approach is especially interesting for complex $\mathrm{CDH}$ cases, where it can help to identify potential novel $\mathrm{CDH}$ causal genes with pleotropic effects.

In this paper, whole exome sequencing was performed on a male fetus diagnosed with $\mathrm{CDH}$ and bilateral congenital cataracts and his two unaffected parents. A likely pathogenic variant was identified in NHS. Pathogenic variants in this gene are responsible for the $\mathrm{X}$-linked Nance-Horan syndrome.

\section{Patients and methods}

\section{Patient history}

The family history is negative for congenital malformations or inherited diseases and the parents are nonconsanguineous. In the index, which is a male fetus, severe right sided $\mathrm{CDH}$ was diagnosed at 25 weeks of gestation. In addition, lenses at both eyes appeared homogeneously echogenic and small, strongly suggesting the diagnosis of bilateral cataracts or lens dysplasia. The baby was born at 38 weeks and deceased on the first day because of a severe pulmonary hypertension. Apgar scores at birth were 3 at $1 \mathrm{~min}, 5$ at $5 \mathrm{~min}$, and 7 at $10 \mathrm{~min}$. Birth weight was $4025 \mathrm{~g}$. Clinical examination excluded any craniofacial 
Table 1 Genetic conditions associating $\mathrm{CDH}$ and congenital ocular defects

\begin{tabular}{|c|c|c|c|}
\hline $\begin{array}{l}\text { Syndrome/genetic } \\
\text { affection }\end{array}$ & Gene & Ocular anomaly & References \\
\hline $\begin{array}{l}\text { Donnai-Barrow } \\
\text { syndrome }\end{array}$ & $L R P 2$ & Iris coloboma/hypoplasia, retinal detachment, severe myopia & {$[6,8-11]$} \\
\hline \multirow[t]{2}{*}{ [MIM 222448] } & & Small optic nerves and chiasm & [12] \\
\hline & & Cataract & {$[6,7]$} \\
\hline $\begin{array}{l}\text { Microphtalmia, } \\
\text { syndromic } 9\end{array}$ & STRA6 & Microphtalmia/anophtalmia & {$[13,14]$} \\
\hline \multicolumn{4}{|l|}{ [MIM 601186] } \\
\hline Goltz syndrome & PORCN & Microphtalmia & {$[15]$} \\
\hline [MIM 305600] & & $\begin{array}{l}\text { Iris and choroid coloboma, } \\
\text { Strabismus }\end{array}$ & \\
\hline $\begin{array}{l}\text { Kabuki syndrome } \\
\text { [MIM 147920] }\end{array}$ & $K M T 2 D$ & Microphtalmia/anophtalmia & [16] \\
\hline $\begin{array}{l}\text { Fryns syndrome } \\
\text { [MIM 229850] }\end{array}$ & $P I G N$ & Microphtalmia/anophtalmia, cloudy cornea & {$[17]$} \\
\hline $\begin{array}{l}\text { Microphtalmia with } \\
\text { linear skin defects }\end{array}$ & $H C C S$ & Microphtalmia, sclecornea, congenital cataract & {$[5]$} \\
\hline [MIM 309801] & & & \\
\hline $\begin{array}{l}\text { Microphtalmia, } \\
\text { syndromic } 6\end{array}$ & BMP4 & $\begin{array}{l}\text { Microphtalmia/anophtalmia, sclerocornea, coloboma, retinal } \\
\text { dystrophy, blepharophimosis, severe myopia? Telecanthus }\end{array}$ & {$[18]$} \\
\hline [MIM 607932] & & & \\
\hline $\begin{array}{l}\text { Microphtalmia, } \\
\text { syndromic } 12\end{array}$ & $R A R B$ & Microphtalmia & [19] \\
\hline [MIM 615524] & & & \\
\hline $\begin{array}{l}\text { Larsen syndrome } \\
\text { [MIM 245600] }\end{array}$ & B3GAT3 & Congenital glaucoma & {$[20]$} \\
\hline
\end{tabular}

dysmorphism. Right $\mathrm{CDH}$ and bilateral congenital nuclear cataracts were confirmed. No other malformations were identified. After obtaining a written informed consent from the couple, the male fetus and both unaffected parents underwent whole exome sequencing.

Following the diagnosis of a maternally inherited NHS variant, the 30-year-old carrier mother underwent an ophthalmologic and stomatologic examination, which ruled out any subclinical abnormality.

\section{Chromosomal microarray analysis}

Copy number profiling was performed using Cytosure syndrome plus $8 \times 60 \mathrm{k}$ arrays (Oxford Gene TechnologyOGT, Oxford, UK) as previously described [21].

\section{Whole exome sequencing}

Following a whole genome library preparation using the TruSeq DNA Library Preparation Kit (Illumina), exome capture was performed with SeqCap EZ Human Exome Library v3.0 (Roche, NimbleGen). Subsequently, 100-bp paired-end reads were generated on Illumina HiSeq2000 according to the manufacturer's instruction.
Reads were mapped to the reference human genome (hg19) using Burrows-Wheeler Aligner 0.6.2. The GATK framework 2.4.9 [22] was used for local realignment, base call recalibration, and SNP calling. Indels were called with Dindel (version 1.01) [23] using default parameters. Variants were annotated with ANNOVAR (version 2013) [24] including for dbSNP (dbSNP137), 1000 genomes data, and the ESP6500 panel (NHLBI GO Exome Sequencing Project (ESP), Seattle, WA) (http://evs.gs.washington.edu/EVS/). Functional predictions for the amino acid changes according to different models (SIFT, Polyphen2, LRT, and MutationTaster) were retrieved from database of human nonsynonymous SNPs and their functional predictions (dbNSFP).

All common variants with a minor allele frequency $>1 \%$ in either 1000 genomes data base, ESP6500 were excluded. In addition, we filtered out non-exonic-site and non-splicesite variants, synonymous variants and in-frame deletions and duplications located in tandem repeat regions. Variants were further filtered based on their frequency in Exome Aggregation Consortium (http://exac.broadinstitute.org/) and in our in-house control group including 338 exomes from unaffected parents and patients with different pathologies, namely, intellectual disability, microcephaly, 
congenital disorder of glycosylation, congenital heart defect, cleft palate, immunopathies, and cancer. Moreover, variants which are predicted to be benign by at least three prediction tools including SIFT, polyphen 2, and mutation taster were ruled out. Finally, relevant variants were identified based on the available genotype-phenotype correlation and on known function of the affected genes. Dominant, recessive, and X-linked models were taken into account for the variants filtering.

PCR followed by Sanger sequencing was performed for variant confirmation. Primers flanking the variant were designed using Primer3Plus web interface (http://www. bioinformatics.nl/primer3plus).

\section{$X$ inactivation assay}

$\mathrm{X}$ inactivation ratios in the carrier mother were identified through X-linked androgen receptor methylation status assessment as previously described [25]. The products were separated on ABI 3730 (Applied Biosystems).

\section{Targeted resequencing}

All the NHS exons with the 20 flanking intronic base pairs, the $3^{\prime}$ and the $5^{\prime}$ untranslated regions were captured using NimbleGen SeqCap target enrichment kit and sequenced on HiSeq2500 platform. Sequencing data analysis and variants filtering strategy are the same as for WES.

\section{RNA extraction and reverse transcription}

Total RNA was extracted from E16.5 fetal mouse diaphragm, lung, heart, and liver. Two C57BL/6J wild type pregnant mice were killed by $\mathrm{CO}_{2}$ exposure. Fetuses were delivered via caesarian section and were killed by decapitation. After laparotomy and thoracotomy, liver, diaphragm, heart, and lungs were isolated under sterile conditions. For each tissue, the total RNA was extracted from four embryos of either litter, using RNeasy Mini kit. Subsequently, $1 \mu \mathrm{g}$ of a pool of three RNA samples in one litter and of two separate RNA samples in the second litter was reverse transcribed with Superscript III using oligo-dt primers.

Two amplicons for NHS were amplified: a $250 \mathrm{bp}$ NHStranscript variant-1-specific fragment with the forward primer ACGTTTAACAGCACCCGTTC and the reverse primer TGATGGGAATGATGCAAGAA; and a $504 \mathrm{bp}$ fragment common to Nhs transcript variant-1 and Nhs transcript variant-2 with the forward primer AGGCTGTGCTGCTCATGTTA and the reverse primer TTAGGAAGTGGCGGTCCTTG (Fig. 1a). As a positive control, we amplified a fragment from GAPDH housekeeping gene using the forward primer
CCTGGAGAAACCTGCCAAGT and the reverse primer TGAAGTCGCAGGAGACAACC. Thirty amplification cycles were performed for all the samples using KAPA HIFI hot start at $65^{\circ} \mathrm{C}$ for Nhs-variant 1 and 2 common fragment and Taq DNA polymerase at $55^{\circ} \mathrm{C}$ for the other ones. The PCR fragments were analyzed on agarose gel.

\section{Literature search for CDH and ocular defects genes}

An automated literature search was conducted on PubMed using the following keywords (congenital diaphragmatic hernia OR cdh OR diaphragmatic defect) AND (congenital ocular defects OR cataract OR anophthalmia OR microphthalmia OR coloboma OR optic OR cryptophthalmos OR aniridia OR glaucoma). Only English papers reporting the combination of $\mathrm{CDH}$ and a congenital eye defect with an available molecular information were taken into account. Further, we retrieved more references using OMIM with the use of same keywords in different combinations.

\section{Submission of variant details to public database}

The NHS variant details and the clinical information of the family are submitted to the Leiden Open Variation Database v3 (https://databases.lovd.nl/shared/genes/NHS).

\section{Results}

\section{NHS loss of function variant probably causes the phenotype}

To determine whether copy number variations might be underlying the developmental defect in the family, a 60k chromosomal microarray was performed. No pathogenic CNVs could be identified. Subsequently, whole exome sequencing was performed for the male fetus and the two unaffected parents. One hundred to one hundred and ten million reads were obtained for the each of the three samples. The mean coverage at each target base varied from $75 \times$ in the fetus to more than $88 \times$ in the parents, with a minimum of $95.8 \%$ of the bases were covered by at least 10 reads. Trio-based variants filtering was performed and resulted in 16 apparent de novo variants in 15 genes, two compound heterozygous genes and three $\mathrm{X}$-linked variants. The list of annotated variants is provided in Table 2. This list was manually curated using functional data and available genotype-phenotype correlation. We first searched for affected genes involved in $\mathrm{CDH}$ and/or in ocular malformations. We identified a maternally inherited stop gain variant chrX:g.17746172C $>\mathrm{T}$ (GRCh37) in NHS gene, which is known to cause $\mathrm{X}$-linked congenital cataract. This NHS missense variant NM_198270.2:c.3883C $>$ T, p. 


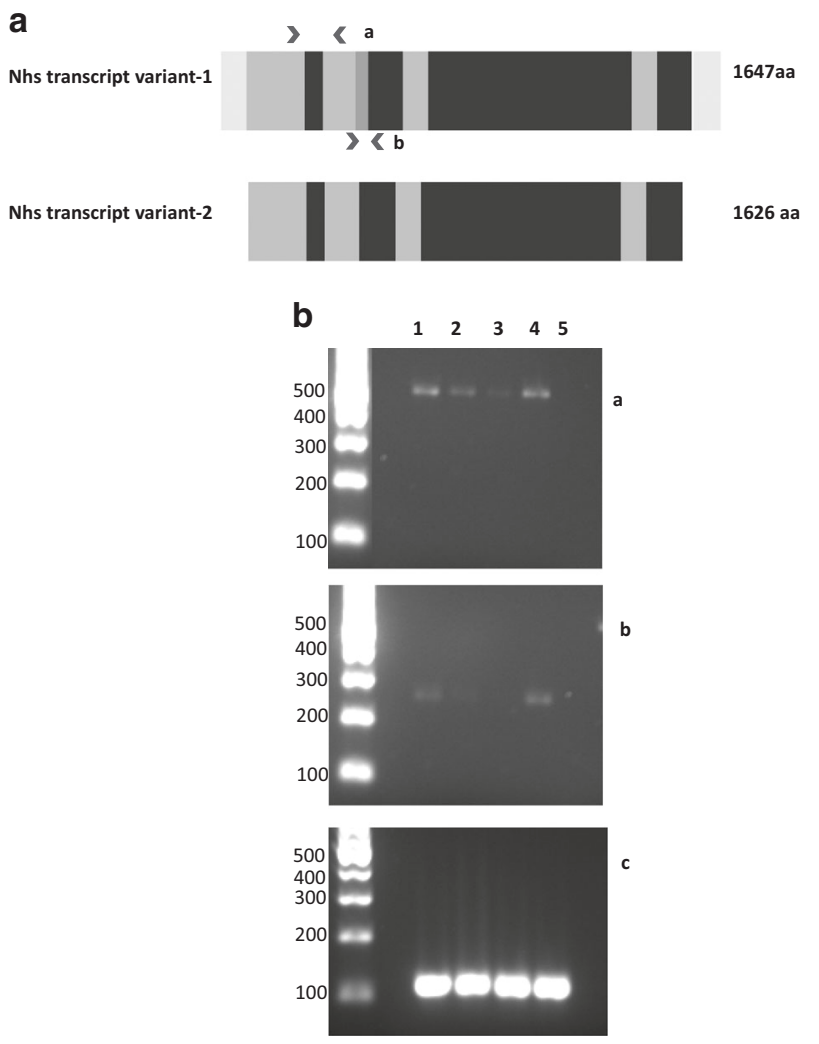

Fig. 1 a Murine NHS gene transcript variants: Nhs transcript variant-2 lacks one alternate in-frame exon, which is highlighted in green and the UTRs. The positions of the primers are shown by red arrows. The Nhs transcript variant- 1 and 2 common amplicon spans from exon 1 to exon 3 (amplicon a). One fragment encompassing exon 4 was amplified for Nhs transcript variant-1 (amplicon b). b Expression analysis of NHS gene. Reverse transcription PCR from E16.5 fetal lung (1), heart (2), liver (3), and diaphragm (4) tissues. The upper panel a shows transcript variant 1 and 2 , the middle panel b shows transcript variant 1 and the lower panel $\mathbf{c}$ shows GAPDH, which was used as a positive control. A negative control (5) is shown for the three PCR reactions. The full colour version of this figure is available at European Journal of Human Genetics online
(Gln1295*) leads to a substitution of a Glutamine by a stop codon and to the loss of the third part of the protein. It is absent in all the public data bases, including GnomAD database, as well as in our in-house control group with a high coverage of the corresponding position. So far 34 pathogenic variants, including both missense and truncating mutations have been reported. Importantly, one stop gain mutation and 9 frameshift deletions located downstream our variant's position, have been reported in Human Gene Mutation Database as a cause of Nance-Horan syndrome. According to the ACMG guidelines [26], these data, in addition to the ocular phenotype, allow to classify the variant as a likely pathogenic variant.

To definitively exclude the implication of other $\mathrm{CDH}$ and ocular defect causing genes, we checked the coverage of genes listed in Table 1, and found a minimum of 20 reads covering all the exons and splice-sites, confirming the reliability of these genes sequencing result. Since $\mathrm{CDH}$ has never been related to $N H S$, we searched for variants in other $\mathrm{CDH}$ candidate genes including inherited variants in known human or mice $\mathrm{CDH}$ genes and genes which are highly expressive in primordial diaphragm. We identified two de novo variants: NM_030632.1:c.6071dup, p.(Pro2025Serfs*23) and NM_001161708.1:c.586C>T, p.(Leu196Phe) in ASXL3 and SYNC, respectively. Both genes are expressed in the primordial murine diaphragm [27].

Truncating mutations in ASXL3 cause Bainbridge-Ropers syndrome associating intellectual disability and distinctive craniofacial dysmorphism. The identified frameshift variant is located at the end of the last exon and is predicted to result in a loss of the last 200 amino acids. This variant is downstream all the reported pathogenic truncating variants, which are predicted to cause a loss of at least 800 amino acids [28]. Given the variant's location and the absence of any facial dysmorphism in the

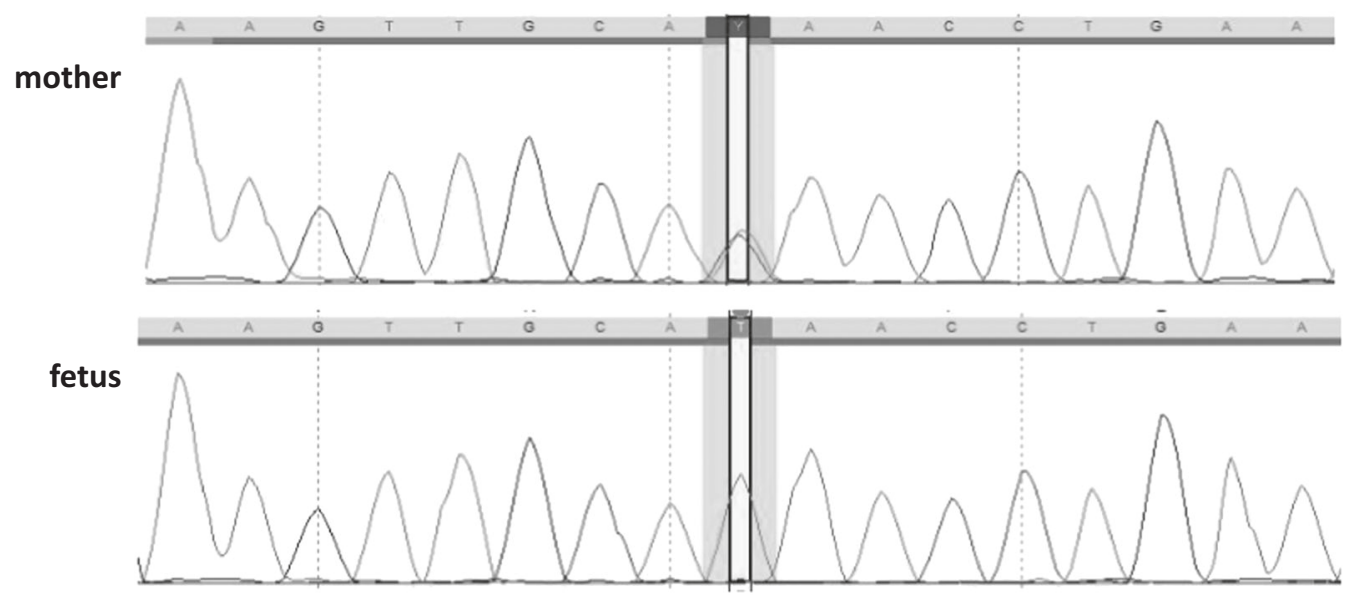

Fig. 2 Sequence chromatogram with $\mathrm{C}$ to $\mathrm{T}$ mutation. The upper panel represents the nucleotide sequence of the carrier mother, and the lower panel represents that of the fetus. The mutation site is highlighted in yellow. The full colour version of this figure is available at European Journal of Human Genetics online 


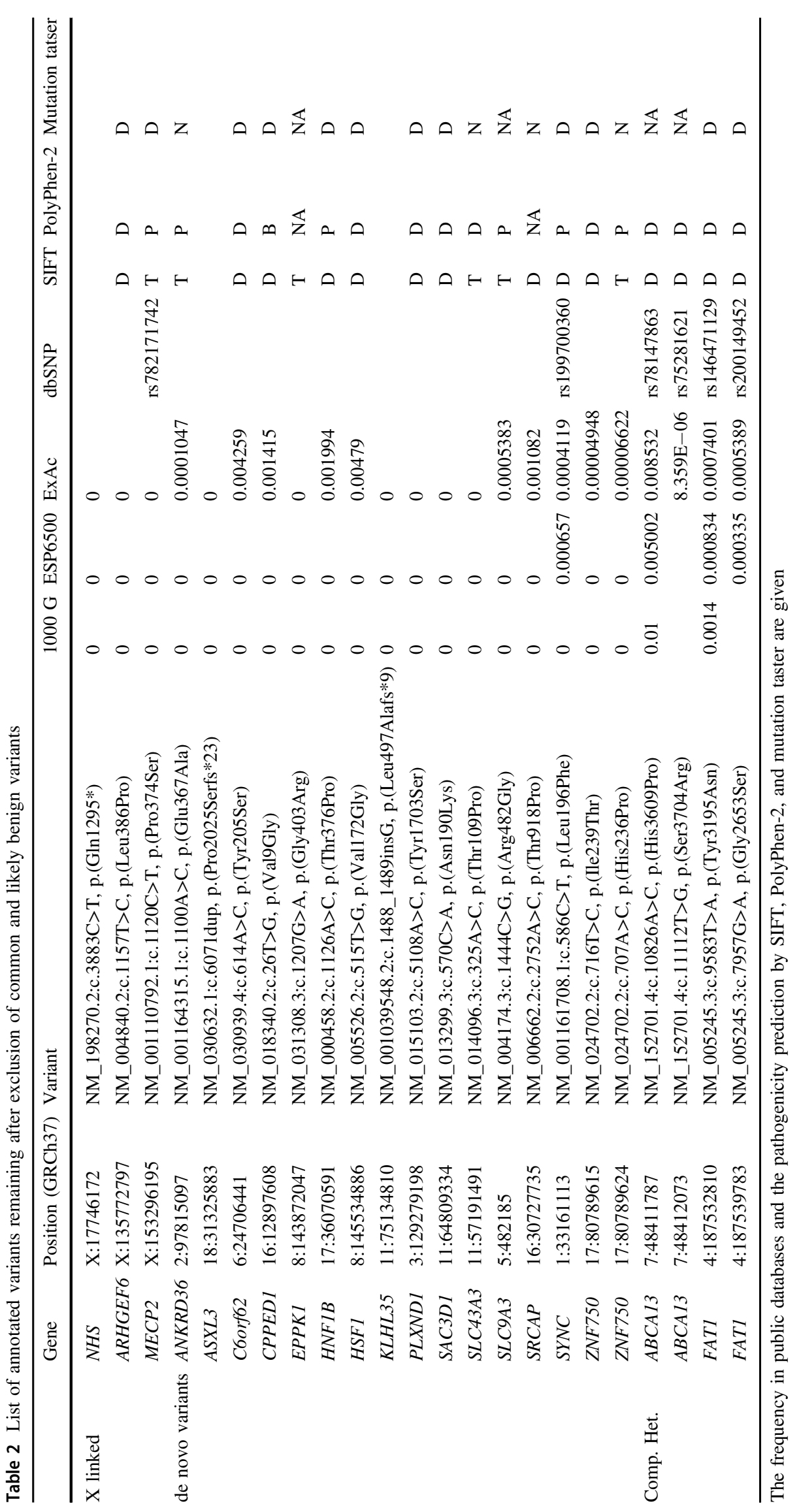


newborn, this variant is categorized as a variant of uncertain significance. $S Y N C$ is highly expressed in primordial diaphragmatic muscle, in cardiac and skeletal muscles. So far, SYNC has never been reported to cause any human pathology. 1p35 deletions encompassing SYNC, reported in Decipher, are not associated with $\mathrm{CDH}$. The identified missense variant is present in dbSNP and in ExAC (allele Number $=9 / 21852$ ). It is predicted to be damaging with a low confidence in SIFT, probably damaging in polyphen 2 and disease causing in Mutation Taster. The variant affects a moderately conserved leucine and substitute it with a phenylalanine that has only small physicochemical differences with the former. We classified this variant as variant of uncertain significance.

To summarize, we identified one NHS likely pathogenic variant and two variants of uncertain significance in $S Y N C$ and ASXL3. We hypothesize that NHS loss of function causes not only congenital cataract but also $\mathrm{CDH}$.

The NHS variant was confirmed by conventional Sanger sequencing in the fetus and in the mother (Fig. 2). As the carrier mother was unaffected, we investigated her $\mathrm{X}$ chromosome inactivation status. This revealed a random inactivation $(56 \%, 44 \%)$.

\section{NHS-targeted resequencing}

To obtain more evidence for the involvement of NHS in $\mathrm{CDH}$, exons, splice-sites, and UTRs were sequenced in 86 probands, fetuses, and neonates, displaying and apparently isolated $\mathrm{CDH}$. No pathogenic variants were identified.

\section{NHS is expressed in the diaphragm}

To determine whether NHS might not only be underlying the bilateral cataract but also the diaphragmatic hernia, we looked whether NHS would be expressed in the developing diaphragm. First, we screened the available expression data in Mouse Genome Informatics data base (http://www. informatics.jax.org). NHS expression is neither documented in diaphragm nor in lung and in developing liver.

Hence, we set out to determine whether NHS might be expressed in the developing diaphragm. RNA was isolated from murine fetal diaphragm at E16.5, date at which the diaphragm is fully developed, in addition to lungs and liver. As a positive control, we used heart tissue where NHS has been shown to be expressed at different embryonic stages. Exon junction PCR on cDNA was performed for GAPDH housekeeping gene, NHS variant1 and NHS total transcript in all four tissues. NHS expression was confirmed in fetal mouse diaphragm, lung and heart at this stage. Compared to GAPDH, NHS has a low expression in all three tissues and very low expression in fetal liver. In heart, transcript variant 1 has a very low expression (Fig. 1b).

\section{Discussion}

The combination of $\mathrm{CDH}$ and congenital cataracts is very rare [5-7]. We describe here the first case of a prenatally detected congenital cataracts in association with $\mathrm{CDH}$, likely caused by a new nonsense variant in NHS gene, which is associated with $\mathrm{X}$-linked Nance-Horan syndrome [MIM. 302350].

Characteristic phenotype of Nance-Horan syndrome includes bilateral severe congenital cataract, distinctive dental anomalies, peculiar dysmorphic features and in $30 \%$ of patients, intellectual disability and behavior disturbance [29, 30]. In addition, other ophthalmological features including microphtalmia, microcornea, and nystagmus [30] and non-ophthalmological finding including brachymetacarpia and variable congenital heart defects were reported in some families [31, 32]. Although $\mathrm{CDH}$ has never been reported in association with $N H S$ gene mutations, one report has described the association of a left $\mathrm{CDH}$ with congenital bilateral cataract in a male patient [33]. In addition, the patient displayed retinal detachment, vitreous hemorrhage, and undescended right testis. Standard karyotyping was normal, but no mutation analysis or exome sequencing was performed [33]. This could be another case of Nance-Horan syndrome with rare ophthalmologic and extra-ocular malformations.

The affected females usually display similar but a less severe phenotype with lens opacity often involving the posterior $Y$ suture $[30,34]$. Since the carrier mother is unaffected and her $\mathrm{X}$ chromosome inactivation profile is random, one could argue that this $N H S$ variant does not affect the protein function. However, given the variant's rarity, nature, and site, and the ocular phenotype of the fetus, which support its pathogenicity, we suggest that the maternal phenotype is rather the result of a reduced penetrance or of a variable onset age of cataract in females. In support of this hypothesis, another asymptomatic female carrier of NM_198270.2:c.1117C $>\mathrm{T}$ variant has been reported [29]. In addition, in a review of Lewi et al. two families with female carriers having a normal ophthalmologic examination have been described [35].

Detailed NHS expression data in humans and in murine fetuses have shown a high and temporally regulated $N H S$ expression in central nervous system, eyes, mainly in lens and at a lower degree in retina, teeth, and heart [36]. These data together with the clinical picture of the syndrome, suggest that NHS is involved in these tissues development. Using reverse transcription, we have shown that NHS has also a potential role in diaphragm development regulation. Three major transcripts have been identified for human NHS with three different transcription start sites in exon 1, exon $1 \mathrm{~A}$ and exon 1B and predicted to encode NHS-A, NHS-1A, and NHS-B isoforms. The two first transcripts are similar to 
the murine Nhs1 and Nhsv1 transcripts, respectively, and are differentially expressed with an exclusive expression of the former in mammalian epithelial cells and of latter in mammalian fibroblasts [36, 37]. Recently, the transcript sequences have been updated with the description of four transcripts variants for human NHS. In mice, two transcripts are available: Nhs transcript variant 1 (NM_001290526) and Nhs transcript variant 2 (NM_001081052.2), predicted to encode, respectively, NHS protein isoform 1 of 1647 amino acid and NHS protein isoform 2 of 1626 amino acid long. These transcripts have similar transcription start site and differ by only one exon (Fig. 1). We show that NHS variant 1 and possibly variant 2 are expressed in the murine diaphragm.

Diaphragmatic muscle is formed from E11.5 to E16.5 in mouse. Recent developmental studies suggest that the diaphragm originates from two major embryonic sources: the pleuroperitoneal folds (PPFs) and the cervical somites derived muscle progenitor cells, which migrate and enter the former by E11.5 [38]. PPFs give rise to the central tendon and to the muscle connective tissue fibroblasts, which have been shown to migrate actively and collectively across the surface of the liver and to control the muscle cells progenitors expansion [38]. We speculate that NHS loss of function causes $\mathrm{CDH}$ through disruption of PPFs cells or of somites-derived cells migration.

NHS has been shown to encode for a functional WAVE homology domain and to be a part of WAVE heteropentameric complex. This complex is critical for actin remodeling and cell morphology control, and subsequently, for cell motility, migration, and cell contact [39]. Other genes previously identified in mouse $\mathrm{CDH}$, such as $\mathrm{GAB} 1$ and EYA1 [40] or in human CDH for instance PTPN12, $T L N 1$, and ARFGEF2 [41] are also involved in cell adhesion and migration, suggesting the importance of this process in diaphragm genesis. Moreover, numerous proteins, including VEGFR1, ROBO1, ROBO3, and CTNNB1, which cause $\mathrm{CDH}$ in mice, were identified as a potential ligand to WAVE complex [42]. Hence, $\mathrm{CDH}$ resulting from one of these genes impairment is possibly due to an abnormal WAVE complex regulation. Further investigations are needed to confirm this hypothesis. In addition, a recent study has constructed human interactome using data from HeLa cells. Thousands of protein interactions have been reported [43]. NHS does not have any interaction with known CDH causal genes. However, it has been reported to bind to TUBG1 and to MLLT1 [40] that are highly expressed in PPFs [27].

To conclude, we report a first case of Nance-Horan syndrome in association with $\mathrm{CDH}$. We suggest that $\mathrm{CDH}$ might be due to NHS loss of function and not a coincidental finding. $\mathrm{CDH}$ in this case is likely to result from an impaired cell migration and cell adhesion. Evidence of involvement of other $\mathrm{CDH}$ genes in this cellular process supports the implication of NHS in diaphragm development. Screening for NHS mutations in unsolved $\mathrm{CDH}$ and cataract cases might confirm NHS as a new $\mathrm{CDH}$ gene.

Acknowledgements We thank UZ Leuven Genomics core team for their technical support and exome sequencing data generation. We thank Enrico Radaelli and Michael Staring from An Zwijsen's team (Department of Cardiovascular Science) for their guidance in mice dissection.

Funding This work was supported by research grants from the KU Leu-ven (SymBioSys [PFV/10/016], GOA/12/015 to J.R.V.), the Herculesfoundation (ZW11-14) and from the Belgian Science Policy OfficeInteruniversity Attraction Poles (BELSPO-IAP) program through the project IAP P7/43-BeMGI. M.K. is supported by the Erasmus+Program of the European Union (Framework agreement number 2013-0040). This publication reflects the views only of the authors, and the European Commission cannot be held responsible for any use that may be made of the information contained therein.

\section{Compliance with ethical standards}

Conflict of interest The authors declare that they have no competing interests.

Ethical approval Approval for biobanked DNA sequencing was granted by KU Leuven/University Hospital Leuven Commission for Medical Ethics S59307. Animal procedures were approved by the ethical committee and performed according to the guidelines of the Animal Welfare Committee of KU Leuven, Belgium (P248/2015).

Informed consent Informed consent was provided to the couple before whole exome sequencing.

\section{References}

1 McGivern MR, Best KE, Rankin J, et al. Epidemiology of congenital diaphragmatic hernia in Europe: a register-based study. Arch Dis Child-Fetal Neonatal. 2014;100:137-44

2 Slavotinek AM. The genetics of common disorders-congenital diaphragmatic hernia. Eur J Med Genet. 2014;57:418-23

3 Pober BR. Overview of epidemiology, genetics, birth defects, and chromosome abnormalities associated with $\mathrm{CDH}$. Am J Med Genet C. $2007 ; 145: 158-71$

4 Stoll C, Alembik Y, Dott B, Roth MP. Associated non diaphragmatic anomalies among cases with congenital diaphragmatic hernia. Genet Counsel. 2014;26:281-98

5 Qidwai K, Pearson DM, Patel GS, et al. Deletions of Xp provide evidence for the role of holocytochrome C-type synthase (HCCS) in congenital diaphragmatic hernia 2010. Am J Med Genet A. 2010;152:1588

6 Kantarci S, Ragge NK, Thomas NS, et al. Donnai-Barrow syndrome (DBS/FOAR) in a child with a homozygous LRP2 mutation due to complete chromosome 2 paternal isodisomy. Am J Med Genet A 2008;146:1842-47.

7 Schowalter DB, Pagon RA, Kalina RE, Mcdonald R. Facio-OculoAcoustico-Renal (FOAR) syndrome. Am J Med Genet 1997;69:45-9.

8 Donnai D, Barrow M. Diaphragmatic hernia, exomphalos, absent corpus callosum, hypertelorism, myopia, and sensorineural 
deafness: a newly recognized autosomal recessive disorder?. Am J Med Genet. 1993;47:679-82

9 Gripp KW, Donnai D, Clericuzio CL, McDonald-McGinn DM, Guttenberg M, Zackai E. Diaphragmatic hernia-exomphaloshypertelorism syndrome: a new case and further evidence of autosomal recessive inheritance. Am J Med Genet. 1997;68:441-4

10 Avunduk AM, Aslan Y, Kapıcıoğlu Z, Elmas R. High myopia, hypertelorism, iris coloboma, exomphalos, absent corpus callosum, and sensorineural deafness: report of a case and further evidence for autosomal recessive inheritance. Acta Ophthalmol Scand. 2000;78:221-2

11 Holmes LB, Schepens CL. Syndrome of ocular and facial anomalies, telecanthus, and deafness. J Pediat. 1972;81:552-5

12 Kantarci S, Al-Gazali L, Hill RS, et al. Mutations in LRP2, which encodes the multiligand receptor megalin, cause Donnai-Barrow and facio-oculo-acoustico-renal syndromes. Nat Genet. 2007;39:957-9

13 Steiner RD, Dignan PSJ, Hopkin RJ, Kozielski R, Bove KE. Combination of diaphragmatic eventration and microphthalmia/ anophthalmia is probably nonrandom. Am $\mathrm{J}$ Med Genet. 2002;108:45-50

14 Pasutto F, Sticht H, Hammersen G, et al. Mutations in STRA6 cause a broad spectrum of malformations including anophthalmia, congenital heart defects, diaphragmatic hernia, alveolar capillary dysplasia, lung hypoplasia, and mental retardation. Am J Med Genet. 2007;80:550-60

15 Brady PD, Van Esch H, Fieremans N, et al. Expanding the phenotypic spectrum of PORCN variants in two males with syndromic microphthalmia. Eur J Hum Genet. 2015;23:551-4

16 McVeigh TP, Banka S, Reardon W. Kabuki syndrome: expanding the phenotype to include microphthalmia and anophthalmia. Clin Dysmorphol. 2015;24:135-9

17 Pierson DM, Taboada E, Butler MG. Eye abnormalities in Fryns syndrome. Am J Med Genet A. 2004;125:273-7

18 Reis LM, Tyler RC, Schilter K. BMP4 loss-of-function mutations in developmental eye disorders including SHORT syndrome. Hum Genet. 2011;130:495-504

19 Srour M, Chitayat D, Caron V, et al. Recessive and dominant mutations in retinoic acid receptor beta in cases with microphthalmia and diaphragmatic hernia. Am J Hum Genet. 2013;93:765-72

20 Jones KL, Schwarze U, Adam MP, et al. A homozygous B3GAT3 mutation causes a severe syndrome with multiple fractures, expanding the phenotype of linkeropathy syndromes. Am J Med Genet Part A. 2015;167:2691-6

21 Brady PD, DeKoninck P, Fryns JP, Devriendt K, Deprest JA, Vermeesch JR. Identification of dosage-sensitive genes in fetuses referred with severe isolated congenital diaphragmatic hernia. Prenat Diagn. 2013;33:1283-92

22 McKenna A, Hanna M, Banks E, et al. The Genome Analysis Toolkit: a MapReduce framework for analyzing next-generation DNA sequencing data. Genome Res. 2010;20:1297-303

23 Albers CA, Lunter G, MacArthur DG, et al. Dindel: accurate indel calls from short-read data. Genome Res. 2011;21:961-73

24 Wang K, Li M, Hakonarson H. ANNOVAR: functional annotation of genetic variants from high-throughput sequencing data. Nucleic Acids Res. 2010;38(16):1-7

25 Allen RC, Zoghbi HY, Moseley AB, Rosenblatt HM, Belmont JW. Methylation of HpaII and HhaI sites near the polymorphic CAG repeat in the human androgen-receptor gene correlates with $\mathrm{X}$ chromosome inactivation. Am J Hum Genet. 1992;51:1229

26 Richards S, Aziz N, Bale S, et al. Standards and guidelines for the interpretation of sequence variants: a joint consensus recommendation of the American College of Medical Genetics and Genomics and the Association for Molecular Pathology. Genet Med. 2015;17:405-23

27 Russell MK, Longoni M, Wells J, Maalouf FI, et al. Congenital diaphragmatic hernia candidate genes derived from embryonic transcriptomes. Proc Natl Acad Sci USA. 2012;109:2978-83

28 Balasubramanian M, Willoughby J, Fry AE, et al. Delineating the phenotypic spectrum of Bainbridge-Ropers syndrome: 12 new patients with de novo, heterozygous, loss-of-function mutations in ASXL3 and review of published literature. J Med Genet 2017;54:537-43. jmedgenet-2016-104360

29 Burdon KP, McKay JD, Sale MM, et al. Mutations in a novel gene, NHS, cause the pleiotropic effects of Nance-Horan syndrome, including severe congenital cataract, dental anomalies, and mental retardation. Am J Hum Genet. 2003;73:1120-30

30 Walpole IR, Hockey A, Nicoll A. The Nance-Horan syndrome. J Med Genet. 1990;27:632-4

31 Coccia M, Brooks SP, Webb TR, et al. X-linked cataract and Nance-Horan syndrome are allelic disorders. Hum Mol Genet. 2009; 18:2643-55

32 Chograni M, Rejeb I, Jemaa LB, Châabouni M, Bouhamed HC. The first missense mutation of NHS gene in a Tunisian family with clinical features of NHS syndrome including cardiac anomaly. Eur J Hum Genet. 2011;19:851-6

33 Kumar P, Ferrone PJ, Fox J, Koppel R. Bilateral cataracts, retinal detachment and vitreous hemorrhage in a newborn with congenital diaphragmatic hernia. J Perinatol. 2003;23:565-566

34 Brooks SP, Ebenezer ND, Poopalasundaram S, Lehmann OJ, Moore AT, Hardcastle AJ. Identification of the gene for NanceHoran syndrome (NHS). J Med Genet. 2004;41:768-71

35 Lewis RA. Mapping the gene for X-linked cataracts and microcornea with facial, dental, and skeletal features to Xp22: an appraisal of the Nance-Horan syndrome. Trans Am Ophthalmol Soc 1989;87:658.

36 Sharma S, Ang SL, Shaw M, Mackey DA, Gécz J, McAvoy JW, Craig JE, Nance-Horan syndrome protein, NHS, associates with epithelial cell junctions. Hum Mol Genet. 2006;15:1972-83

37 Sharma S, Koh KS, Collin C, et al. NHS-A isoform of the NHS gene is a novel interactor of ZO-1. Experimental cell Res. 2009;315:2358-72

38 Merrell AJ, Ellis BJ, Fox ZD, Lawson JA, Weiss JA, Kardon G. Muscle connective tissue controls development of the diaphragm and is a source of congenital diaphragmatic hernias. Nat Genet. 2015;47:496-504

39 Brooks SP, Coccia M, Tang HR, et al. The Nance-Horan syndrome protein encodes a functional WAVE homology domain (WHD) and is important for co-ordinating actin remodelling and maintaining cell morphology. Hum Mol Genet. 2010;19:2421-32

40 Longoni M, High FA, Russell MK, et al. Molecular pathogenesis of congenital diaphragmatic hernia revealed by exome sequencing, developmental data, and bioinformatics. Proc Natl Acad Sci USA. 2014;111:12450-5

$41 \mathrm{Yu} \mathrm{L}$, Sawle AD, Wynn J, Aspelund G, et al. Increased burden of de novo predicted deleterious variants in complex congenital diaphragmatic hernia. Hum Mol Genet. 2015;24:4764-73

42 Chen B, Brinkmann K, Chen Z, et al. The WAVE regulatory complex links diverse receptors to the actin cytoskeleton. Cell. 2014;156:195-207

43 Hein MY, Hubner NC, Poser I, et al. A human interactome in three quantitative dimensions organized by stoichiometries and abundances. Cell. 2015;163:712-23 